\title{
Computed tomography artefacts: An experimental investigation of causative factors
}

\author{
N Strumas DDS MD, O Antonyshyn MD FRCSC, MJ Yaffe BSc MSc PhD, G Mawdsley BSc FCCPM, \\ P Cooper MD FRCSPC \\ Division of Plastic Surgery; Imaging Research; and Department of Neuroradiology, Sunnybrook Health \\ Science Centre; and Departments of Medical Biophysics and Medical Imaging, University of Toronto, \\ Toronto, Ontario
}

\begin{abstract}
N Strumas, O Antonyshyn, MJ Yaffe, G Mawdsley, P Cooper. Computed tomography artefacts: An experimental investigation of causative factors. Can J Plast Surg 1998;6(1):23-29.

The factors that produce computed tomography (CT) artefacts associated with craniofacial fixation devices were analyzed. The effects of attenuation, motion, partial volume and implant shape were evaluated. By using a CT phantom model with an engine to produce reproducible motion, a vitallium rod, fixation plate and blank were scanned sequentially. For each experimental condition, the degree of artefact produced was quantified by measuring the standard deviation of the CT number at standardized regions of interest, and a comparative analysis was performed. Motion produced the greatest degree of artefact. Both the frequency and direction of motion were important, with high frequency motion in the plane of the CT slice producing the greatest degree of CT artefact. Partial volume artefacts increased as the volume of material in the plane of section increased. The amount of artefact produced was dependent on the volume of material x-rays passed through the object rather than on the geometric configuration. Attenuation effects were evaluated. Results revealed that the amount of artefact was greatest between highly attenuating objects.
\end{abstract}

Key Words: Computed tomography artefacts, Craniofacial fixation devices

\section{Interférences à la tomographie par ordinateur : recherche expérimentale des facteurs responsables}

RÉSUMÉ : Les facteurs qui entraînent les interférences à la tomographie par ordinateur associées aux appareils de fixation cranio-faciaux ont été analysés. Les effets de l'atténuation du mouvement, du volume partiel et de la taille de l'implant ont été évalués. À l'aide d'un modèle de CT factice doté d'un moteur apte à produire, de façon reproductible, le mouvement, la tige de vitallium et la plaque de fixation ont été scanographiés en séquence. Pour chacune des conditions expérimentales, le degré d'interférence produit a été mesuré au moyen de déviations standards en certains points d'intérêt et une analyse comparative a été effectuée. Le mouvement a produit le degré le plus élevé d'interférences. La fréquence et la direction du mouvement ont toutes deux été importantes, les mouvements de haute fréquence du plan produisant le degré le plus élevé d'interférences. Les interférences de volume partiel ont augmenté à mesure que le volume de matériel inclus dans le plan de la section augmentait. La quantité d'interférences produite dépendait du volume de matériel traversé par les rayons X plutôt que de sa configuration géométrique. Les effets d'atténuation ont été mesurés; les résultats ont révélé que la quantité d'interférences était plus forte entre les objets hautement atténués.

$\mathrm{M}$ etallic internal fixation devices are employed routinely in craniofacial surgery to provide three-dimensional stability and facilitate the bony union of skeletal segments. However, these fixation devices produce artefacts in computed tomography (CT) scans, which can potentially interfere with the diagnostic interpretation of postoperative radiographical images. CT artefacts are recognized as a significant

This paper was presented at the 49th Annual Meeting of the Canadian Society of Plastic Surgeons, Saskatoon, Saskatchewan, June 1995

Correspondence and reprints: Dr Oleh Antonyshyn, Division of Plastic Surgery, H-271, Sunnybrook Hospital, 2075 Bayview Avenue, Toronto, Ontario M4N 3M5. Telephone 416-480-4868, fax 416-480-6800, e-mail oantonys@globalserve.net clinical problem associated with the use of hip arthroplasties $(1,2)$, spinal fixation devices $(3,4)$, intracranial clips $(5)$ and intra-abdominal clips $(6,7)$. Recently, Fiala et al (8) and Sullivan et al (9) independently investigated CT artefacts generated by craniofacial fixation devices, and specifically described the degrees of artefact associated with variations in size and alloy composition of implants. Anastakis et al (10) further analyzed the degree to which CT artefacts interfere with the visualization of craniofacial anatomy. Titanium implants of any size and microvitallium implants did not produce significant image degradation, while larger vitallium implants interfered with the visualization of anatomy, particularly of soft tissue windows and in the immediate vicinity of the implant (10). 

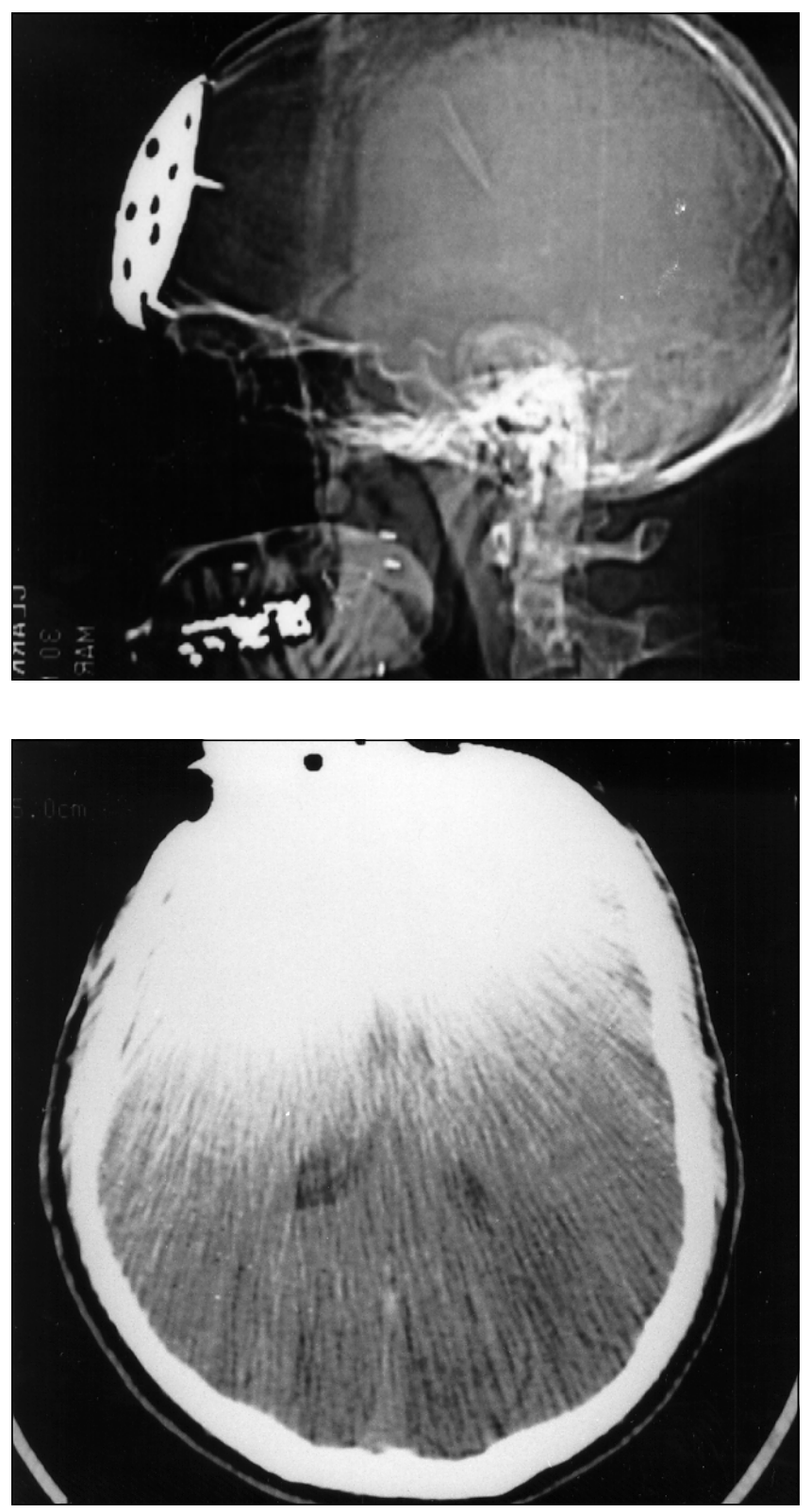

Figure 1) Top Plain x-ray of a lateral skull with a perforated tantalum plate previously employed in cranioplasty for a post-traumatic frontal defect. Bottom Axial computed tomography of the soft tissue window. The artefact produced by the tantalum plate interferes with visualization of the frontal sinus and intracranial soft tissue

These findings provide clinically relevant guidelines for the craniofacial surgeon. In situations where postoperative $\mathrm{CT}$ imaging is important, ie, following tumour ablative procedures or cranio-orbital procedures, image degradation is minimized by the intraoperative use of the least attenuating fixation devices of the smallest calibre and number possible to achieve stability of bone segments. However, there is a large population of patients with larger calibre or highly attenuating craniofacial implants in whom diagnostic interpretation of CT images is significantly compromised (Figure 1). Minimizing the degree of artefact is predicated on having a thorough understanding of the physics of CT artefacts and of
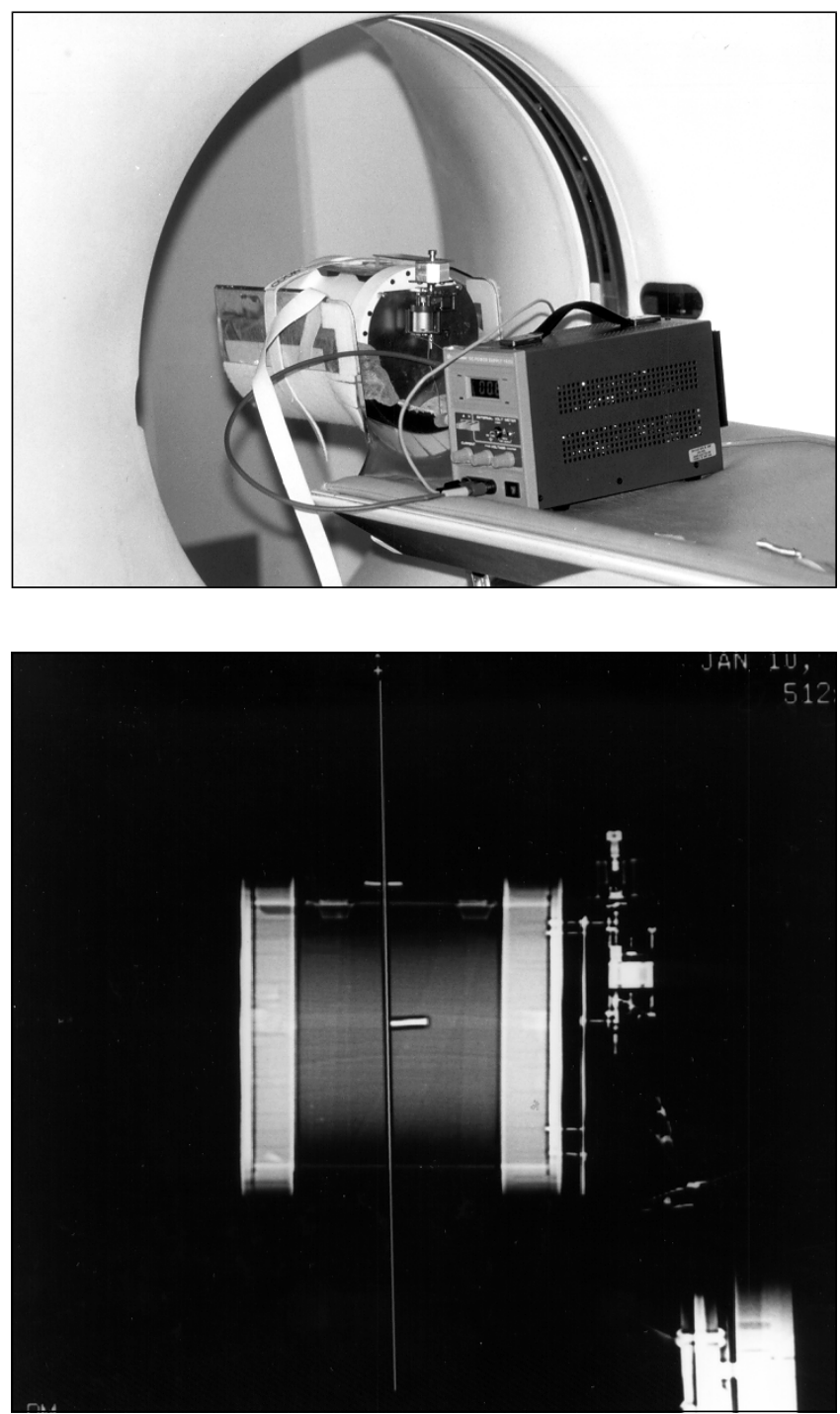

Figure 2) Top Teflon cylinder filled with distilled water is positioned within the computed tomography scanner. An engine, shown in front of the phantom, produced reproducible motion of the platform on which implants were placed for scanning. Bottom Scout x-ray demonstrating the plane of axial computed tomography relative to the phantom implant

the relative contributions of the various factors that cause image degradation.

A CT phantom model was employed to analyze the factors that produce CT artefacts associated with craniofacial fixation devices. The effects of attenuation, implant shape, partial volume and motion were specifically evaluated.

\section{MATERIALS AND METHODS}

A CT phantom was constructed for this experiment (Figure 2). The CT phantom consisted of a tetrafluoroethylene-fluorocarbon (Teflon, Du Pont, Delaware) cylinder measuring $20 \mathrm{~cm}$ in diameter with a wall thickness of $0.5 \mathrm{~cm}$. The Teflon shell had a CT number approximating that of cortical bone. Three plastic radiographically tissue-equivalent rods with different compositions were placed in the phantom. These rods served as internal controls and had densities equivalent 


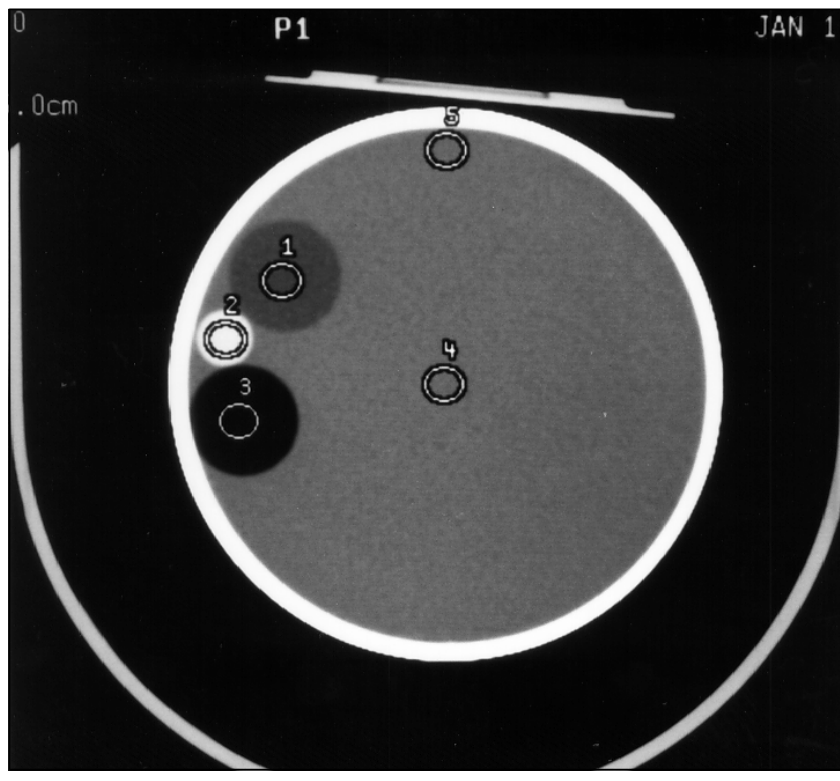

Figure 3) Computed tomography scan evaluation technique. Regions of interest (ROIs) 1, 2 and 3 serve as internal controls and represent densities of the globe, intraorbital muscle and fat. ROI 4 is located in the centre of the phantom, and ROI 5 is immediately beneath the platform on which specific implants are evaluated. PI Vitallium plate

to fat, muscle and the ocular globe. The phantom was filled with distilled water. A vitallium rod was secured to the side of the phantom with its long axis parallel to the cylinder to enhance attenuation of the object being studied. The internal fixation devices were secured to a platform on the external surface of the phantom with their longitudinal axes parallel to the axis of the phantom.

To evaluate the effect of motion on CT artefacts, an engine that allowed for the production of reproducible motion was constructed. This engine moved the platform on which the implant was mounted at a specified frequency and amplitude. This mechanism further controlled the direction of motion, ie, within the plane of the CT scan or perpendicular to the scan plane.

Different implant parameters were analyzed by using three different implants - a vitallium rod measuring $2 \times 0.31 \mathrm{~cm}$, a four-hole vitallium plate and a blank (a vitallium plate of similar dimensions but without fixation holes).

\section{Scanning protocol}

The CT scanning protocol was standardized, and routine technical parameters for clinical craniofacial imaging (voltage $120 \mathrm{kV}$, tube load $200 \mathrm{~mA} \times 2 \mathrm{~s}, 3.0 \mathrm{~mm}$ slices, gantry tilt 0 ) were employed.

\section{Experimental conditions}

The first experiment evaluated the effect of attenuation on $\mathrm{CT}$ artefacts. A vitallium rod was placed on the CT phantom platform with its long axis parallel to that of the phantom. Attenuation was analyzed by quantifying the degree of artefact generated by a single implant and by two implants. The second experiment evaluated partial volume effects by using a
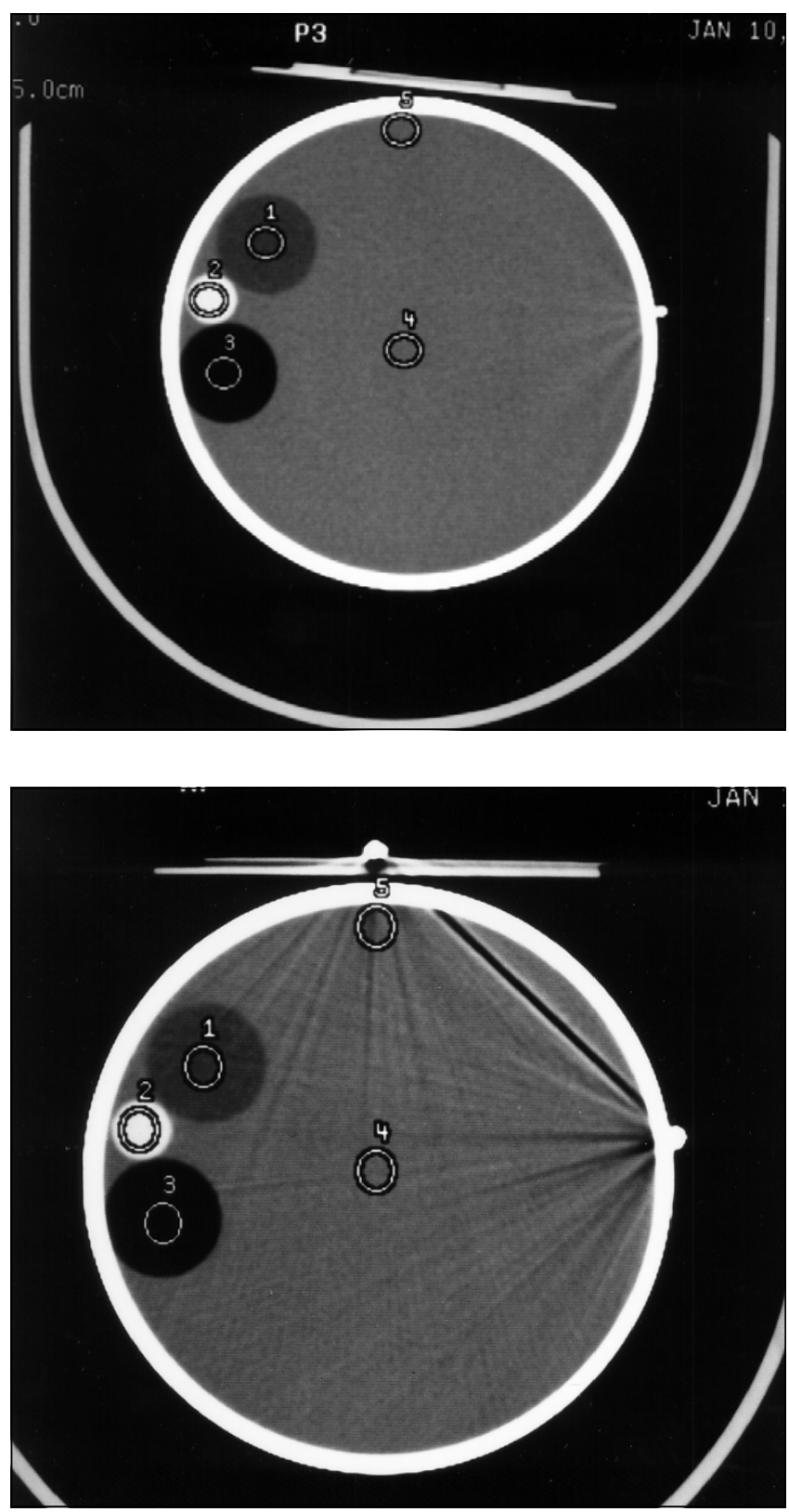

Figure 4) Attenuation effect. Top A single implant (on the right side of the phantom) produced a localized streak artefact emanating from the implant. Bottom Two implants substantially increased the amount of artefact, particularly between objects. P3 Vitallium rod

single vitallium rod and sequentially increasing the amount of rod present within the scan. The third experiment analyzed the effects of implant shape by comparing the degree of artefact generated by a vitallium four-hole fixation plate and that of a blank plate of similar dimensions. The fourth experiment analyzed the effects of motion with respect to direction and frequency.

\section{Evaluation of CT scans}

Regions of interest (ROI) were selected in areas of uniform composition such that noise could only be attributed to quantum statistics and system noise. An increase in standard de- 


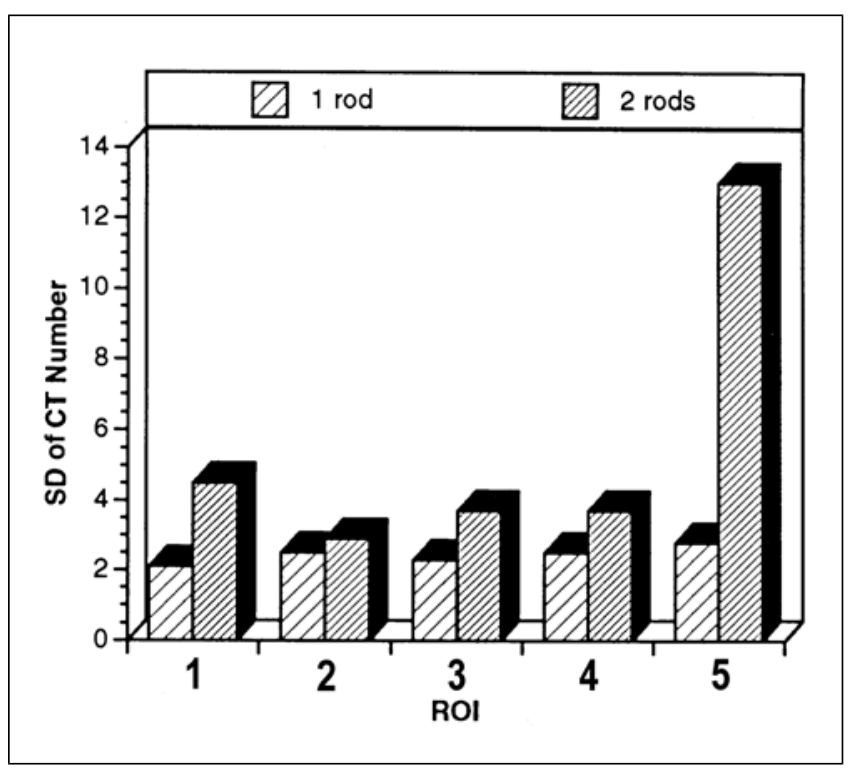

Figure 5) Effect of attenuation on computed tomography (CT) artefacts. ROI Region of interest; SD Standard deviation

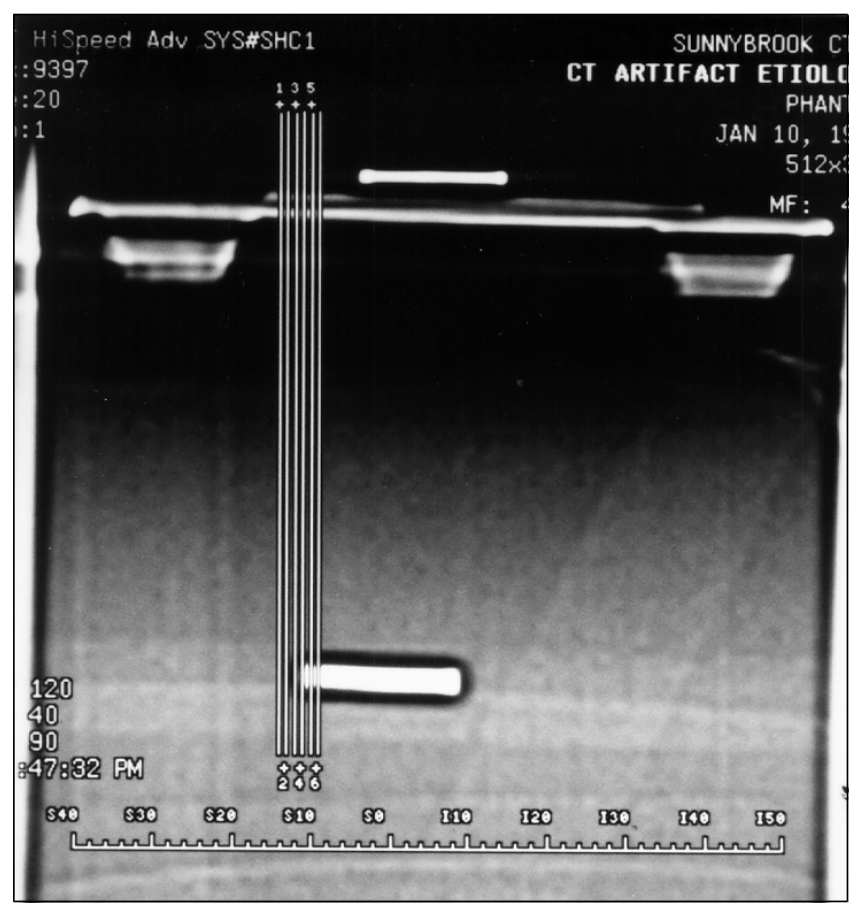

Figure 6) Technique of assessing partial volume effect. The implant was advanced into the plane of the scan to present increasing volumes of attenuating material within a computed tomography $(C T)$ slice

viation in the number of ROIs was related to the inability of the scanner software to correctly assign CT numbers to a pixel due to nonlinearities, beam hardening or other data inconsistencies.

Five standardized ROIs were identified within an axial projection of the CT phantom (Figure 3). The size and location of these ROIs were standardized for each scan. ROIs 1, 2 and 3 served as internal controls representing densities of intraorbital soft tissue. ROI 4 was located within the centre of

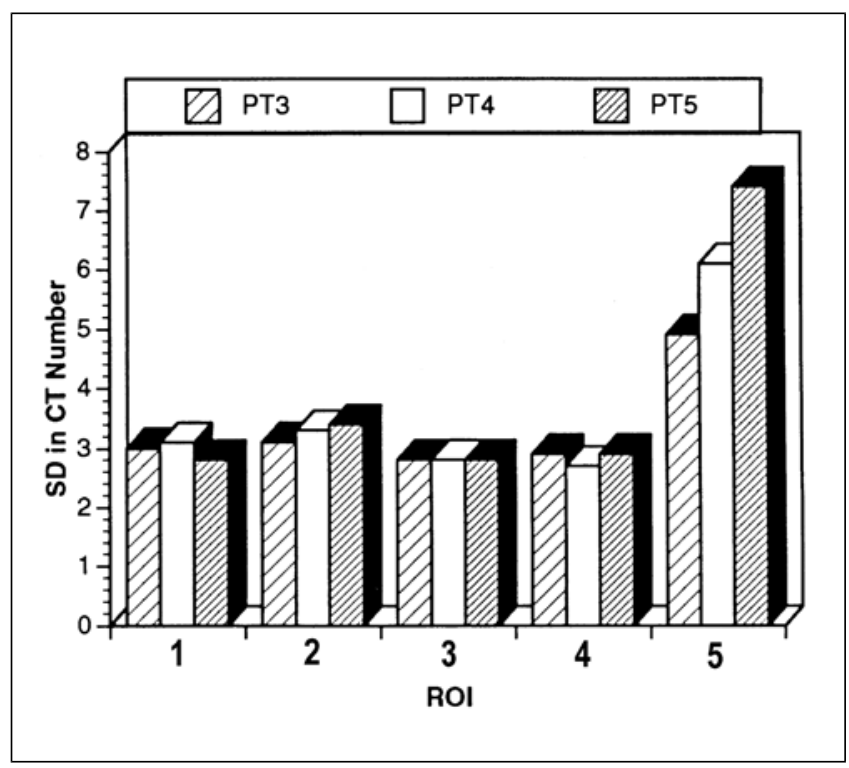

Figure 7) Effect of partial volume on computed tomography (CT) artefacts. Increasing volumes of implant within a CT slice were used (partial thickness [PT] 3 of $9 \mathrm{~mm}$, PT4 of $12 \mathrm{~mm}$ and PT5 of $15 \mathrm{~mm}$ ). ROI Region of interest; SD Standard deviation

the phantom, and ROI 5 was located immediately beneath the implant. The CT number in Hounsfield units (ie, the relative $\mathrm{x}$-ray absorption coefficient) was calculated for each ROI, and the standard deviation of the CT number was calculated. The standard deviation of the CT number is a direct indication of the variation in CT number within a ROI and provides a quantitative indication of the magnitude of the artefact produced.

\section{Effect of attenuation}

\section{RESULTS}

The presence of a single metal implant (attenuating object) within an axial CT slice produced an artefact characterized by radiolucent and radiodense streaks emanating from the object (Figure 4A). The presence of two attenuating objects within a CT scan slice significantly increased the degree of artefact, and resulted in image degradation and streaking, particularly between the two objects (Figure 4B). Quantitative analysis revealed an increase in the SD of the CT number in all ROIs when two implants were present, particularly in ROI 5, which was adjacent to the implant (Figure 5).

\section{Partial volume effect}

Partial volume effects were studied by sequentially increasing the amount of implant present within the scan (Figure 6). The degree of artefact produced, particularly in ROI 5, increased as the volume of material in the plane of section increased (Figure 7).

\section{Implant shape}

The effect of implant shape on CT artefact was analyzed by comparing two implants identical in shape and dimension, differing only in the presence or absence of screw fixation 


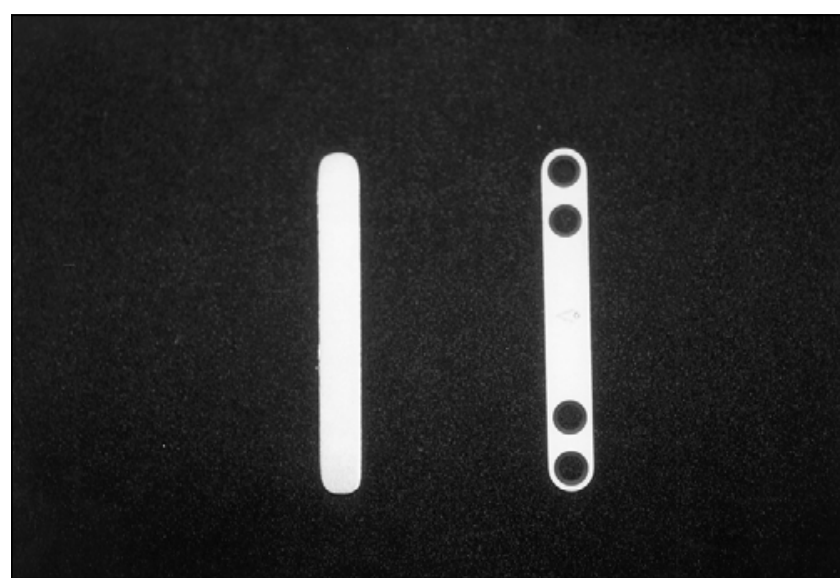

Figure 8) Vitallium blank plate (left) and four-hole fixation plate used to evaluate the effect of implant shape on computed tomography artefacts

holes (Figure 8). The presence of holes in a fixation plate may produce inconsistencies in the scanned image. However, in this experiment, the fixation plate with holes consistently produced fewer artefacts than the comparable blank implant across all ROIs (Figure 9).

\section{Effective motion}

Motion produced obvious CT image degradation (Figure 10). For a given fixed amplitude and frequency, motion in the axial plane (ie, in the plane of the CT scan slice) produced the greatest standard deviation of the CT number across all ROIs, particularly in ROI 5 (Figure 11). With motion maintained at a fixed amplitude in the axial plane, a significant number of artefacts were noted at a frequency of $0.5 \mathrm{~Hz}$, and the number increased significantly with an increase in frequency to $5 \mathrm{~Hz}$ (Figure 12).

When all potential CT artefact-generating effects were compared, the greatest variation in the standard deviation of the CT number was produced by motion in an axial plane (Figure 13).

\section{DISCUSSION}

When an object is scanned by CT, $\mathrm{x}$-ray radiation is received from many different angles and passes through each point of the object. The image produced is a result of computer reconstruction of all relative attenuation values at each point. The reconstruction program assumes that all measurements are internally consistent. Any inconsistency results in artefacts in the final image (6).

Objects with high attenuation coefficients (high atomic number) result in beam hardening (11), which occurs due to the polyenergetic nature of the $\mathrm{x}$-ray beam used in the scanner. As the beam passes through a material, more low energy $\mathrm{x}$-rays are attenuated from the beam than high energy $\mathrm{x}$-rays, resulting in a beam that is 'harder' and composed of a greater proportion of high energy $\mathrm{x}$-rays. This beam has different attenuation characteristics than the original beam. During reconstruction, it is assumed that the beam is monoenergetic and experiences uniform attenuation through a homogenous

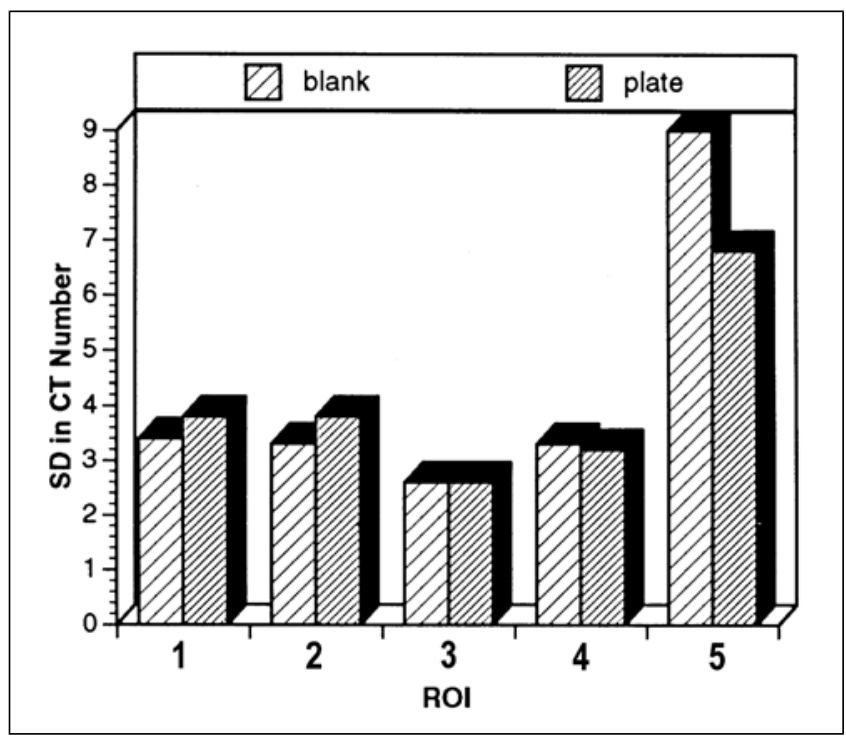

Figure 9) Effect of implant shape on computed tomography (CT) artefacts. ROI Region of interest; SD Standard deviation

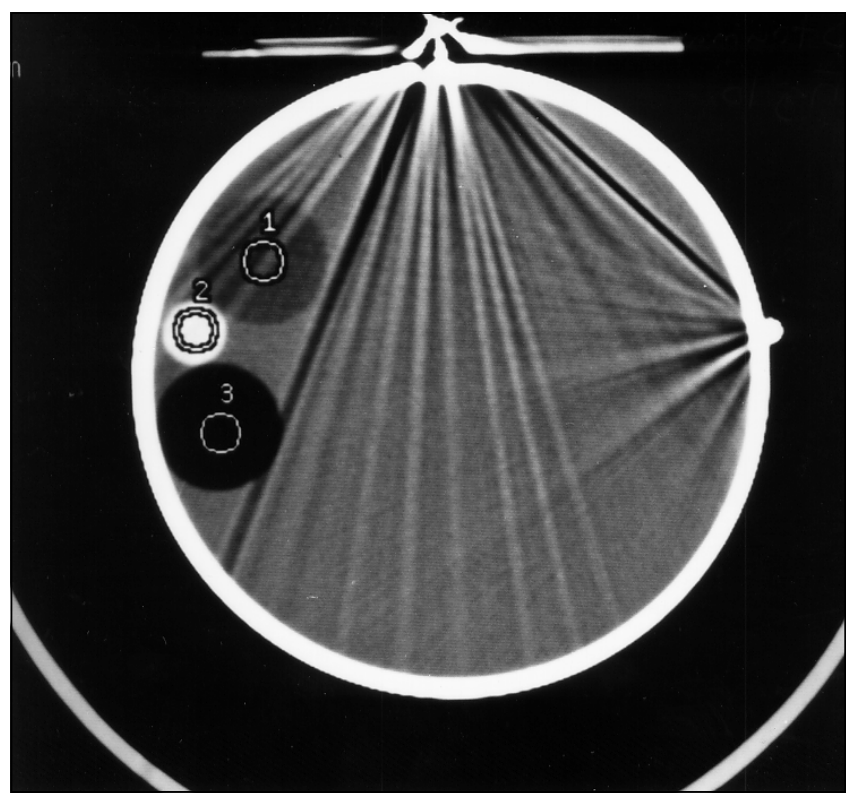

Figure 10) Axial phantom computed tomography scan demonstrating the increase in artefacts with minimal motion $(0.5 \mathrm{~Hz})$ in the axial plane

medium. This results in a nonlinear response to thickness changes. With highly attenuating materials, the transmitted radiation levels, which are near the minimum signal and noise limits of the electronic detectors, are attenuated. Depending on the electronic design, truncation of values or removal of offsets may result in signals with ' 0 ' values. As a result, projection data through long path lengths of highly attenuating material may result in signals that are incorrect, which result in inconsistencies in the calculation of the image.

A number of factors have been shown to generate inconsistent projection data (12). Objects with high attenuation coefficients do not allow complete penetration by the x-ray 


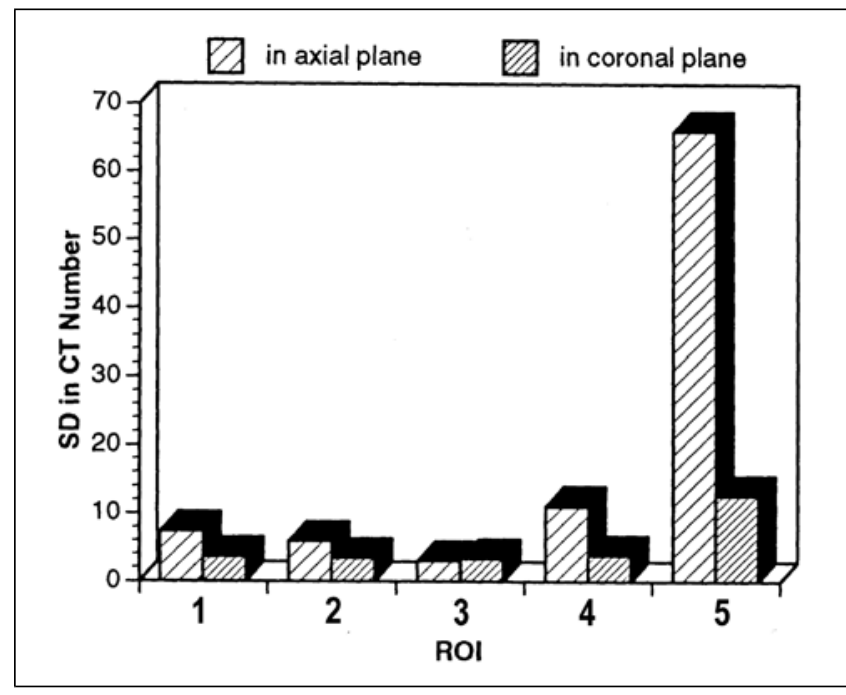

Figure 11) Effect of motion direction at $5 \mathrm{~Hz}$ on computed tomography (CT) artefacts. ROI Region of interest; SD Standard deviation

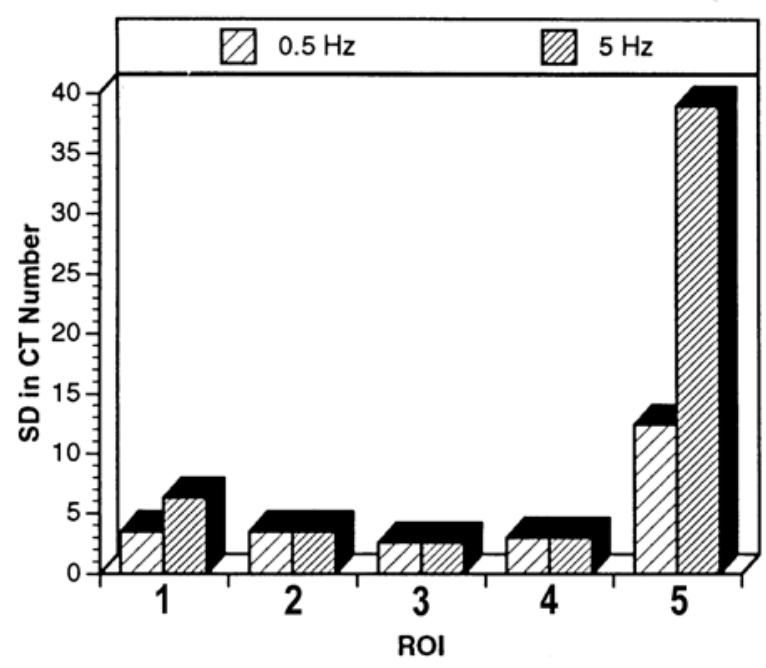

Figure 12) Effect of motion frequency in the axial plane on computed tomography (CT) artefacts. ROI Region of interest; SD Standard deviation

beam. As a result, the detector receives a decreased signal, resulting in missing projection data. Reconstructions using this data exhibit low density streak artefacts emanating from the object (6). The number of streak artefacts increases with increasing attenuation coefficient and material path lengths $(1,2)$. This relationship is expressed by Beer's law:

$$
I=I_{0} e^{-t \mu}
$$

where an $\mathrm{x}$-ray beam with an initial intensity, $\mathrm{I}_{0}$, has an intensity of I after passing through a material of thickness $t$ with an attenuation coefficient of $\mu$ (8).

An increased number of artefacts was associated with greater attenuation and longer path lengths of the material. A greater number of streak or 'starburst' artefacts was seen emanating from the implants when a larger material path

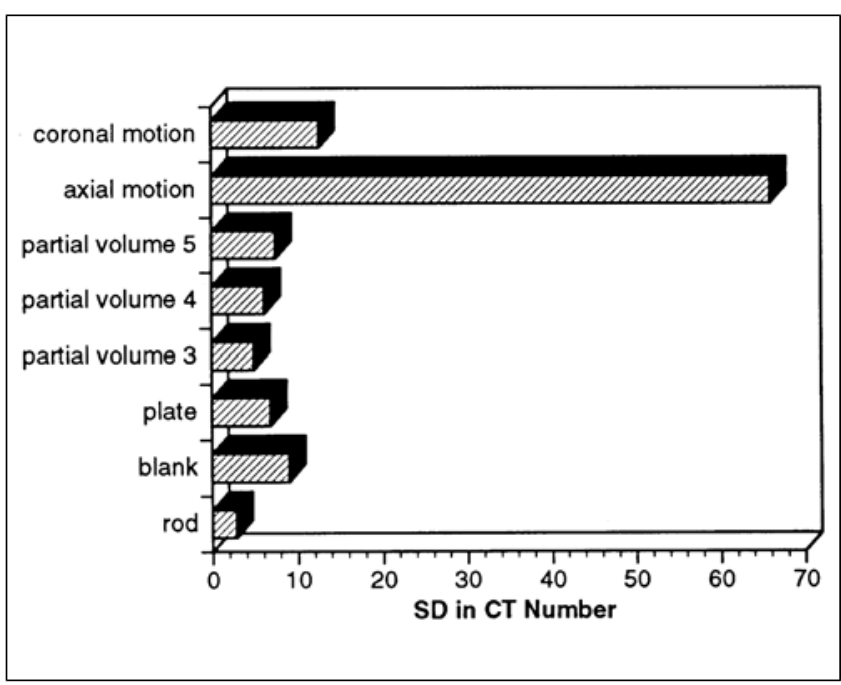

Figure 13) Graph comparing the causes of computed tomography (CT) artefacts. SD Standard deviation

length was used. The greatest degree of artefact was detected in the RIO immediately adjacent to the implant. When two highly attenuating objects were present, streak artefacts were most prominent between the two objects, resulting from an increase in the total path length of highly attenuating material, as well as from an exacerbation of the beam hardening and signal reduction problem.

The effect of shape was evaluated to determine whether the angles present on a fixation plate contributed to an increase in the degree of artefact and whether empty screw holes contributed to a partial volume effect. However, no significant effects were produced by varying the shape of the object. The volume rather than the geometric configuration of the material was the main factor in determining the degree of artefact generated.

Partial volume effects cause artefacts when objects are axially nonuniform in the field of view (13). Any axial variation in attenuation results in inconsistencies in the data set used in the reconstruction algorithm, which results in streak artefacts. Partial volume effects may occur in the axial or transverse directions; however, the greater effect is seen in the axial dimension. In a CT scanner, a larger volume is sampled in the axial dimension than in the transverse direction. Also, transverse partial volume effects within a slice vary from view to view, so they may be averaged out during reconstruction, whereas axial effects are consistent from view to view (13). The effect of partial volume was significant. In particular, more artefacts were produced as the volume of material in the plane of section increased. This is consistent with what is known about partial volume effects.

The degree of CT artefact is particularly enhanced by motion of the object. Subtle motion of a high density object results in streaking (12). The slight motion produced by respiratory, circulatory or peristaltic functions is sufficient to produce streak artefacts (6). Motion was evaluated specifically in this study and produced the greatest degree of artefact. In particular, horizontal motion within the plane of the 
CT scan produced the greatest degree of artefact, and this effect increased with the frequency of motion.

Artefacts caused by motion result when an object occupies different partial volumes during each acquisition of projection data during the scan. This causes inconsistencies in the data set used for image reconstruction and, hence, the production of artefacts.

\section{CLINICAL IMPLICATIONS}

The results of this study have direct clinical implications, providing guiding principles for the intraoperative use of craniofacial metal implants to minimize subsequent CT artefacts and for postoperative CT scanning to optimize quality despite the presence of implants.

The present study showed that attenuation of the x-ray beam by a metallic object is a consistent cause of CT artefacts. The degree of attenuation is proportional to the volume or size of the implant and the atomic numbers of the components of the alloys used to construct the implant. This study specifically demonstrated that implant shape does not affect the degree of CT artefact. Furthermore, the presence of two implants within a single CT slice greatly potentiates the degree of attenuation (ie, the observed CT artefact and standard deviation of the CT number are far greater than the those of the sum of the effects of two individual implants).

Practical implications for intraoperative planning are as follows.

- Implant shape is not important.

- Implants of the smallest possible size should be employed.

\section{REFERENCES}

1. Fishman EK, Magid D, Robertson DD, Brooker AF, Weiss P, Siegelman SS. Metallic hip implants: CT with multiplanar reconstruction. Radiology 1986;160:675-81.

2. Robertson DD, Weiss PJ, Fishman EK, Magid D, Walker PS. Evaluation of CT techniques for reducing artifacts in the presence of metallic orthopaedic implants. J Comput Assist Tomogr 1988;12:236-41.

3. Dietrich U, Kalff R, Stürmer KM, Serdarevic M, Kocks W. Computerized tomography after internal fixation of the spine. Neurosurg Rev 1989;12:211-5.

4. Citrin CM. Multi-planar reconstruction as a method of eliminating streak artifact in computed tomographic images. Comput Radiol 1982;6:377-8.

5. Maciunas RJ, Juneau P. Limiting artifact in CT stereotaxic periventricular procedures. J Neurosurg 1988;69:459-60.

6. Marks WM, Callen PW. Computed tomography in the evaluation of patients with surgical clips. Surg Gynecol Obstet 1980;151:557-8.

7. Saxe AW, Doppman JL, Brennan MF. Use of titanium surgical clips to avoid artifacts seen on computed tomography. Arch Surg 1982;117:978-9.
- The greatest degree of artefact and image degradation is in the region immediately adjacent to the implant. Some thought should be given to avoid implanting immediately adjacent to an area that might have to be followed very closely radiographically in the postoperative period.

- Most importantly, the smallest number of implants should be employed, and preferably these should not be placed in the same axial or coronal plane.

Methods aimed at decreasing or eliminating artefacts from scans have been developed. Because an artefact is the result of inconsistent image data, reconstruction algorithms have been developed for decreasing or removing artefacts. However, these programs are not widely available $(12,14,15)$. Other proposed methods include reformatting the image into new orthogonal or oblique images $(1,4)$. However, these methods are difficult to employ in craniofacial fixation because multiple plates are being used in multiple locations with different axes of orientation.

There are additional direct and practical implications that optimize the quality of CT scans in a patient with craniofacial devices or implants in place. Motion is the factor that most significantly affects the degree of artefact. This occurs in a physiological range, ie, with a minimum rate of motion less than 60 cycles per $\mathrm{s}$. This effect is even more dramatic with gross movements of the head during scanning. The most important factors in obtaining optimal scans are providing better head holders to minimize gross head motion and employing the fastest possible scan rates (eg, helical scanners are likely to produce better image quality).

8. Fiala TGS, Novelline RA, Yaremchuk MJ. Comparison of CT imaging artifacts from craniomaxillofacial internal fixation devices. Plast Reconstr Surg 1993;92:1227-32.

9. Sullivan PK, Smith JF, Rozzelle AA. Cranio-orbital reconstruction: safety and image quality of metallic implants on CT and MRI scanning. Plast Reconstr Surg 1994;94:589-96.

10. Anastakis DJ, Antonyshyn OM, Cooper PW, Yaffe MJ, Bush K, Mawdsley GE. Computed tomography artifacts associated with craniofacial fixation devices: an experimental study. Ann Plast Surg 1996;37:349-55.

11. Rao PS, Alfidi RJ. The enviromental density artifact: A beamhardening effect in computed tomography. Radiology 1981;141:223-7.

12. Glover GH, Pelc NJ. An algorithm for the reduction of metal clip artifacts in CT reconstruction. Med Phys 1981;8:799-807.

13. Glover GH, Pelc NJ. Nonlinear partial volume artifacts in x-ray computed tomography. Med Phys 1980;7:238-47.

14. Morin RL, Raeside DE. Removal of streaking artifact in computed tomography. J Med Systems 1982;6:387-97.

15. Kalender WA, Hebel R, Ebersberger J. Reduction of CT artifacts caused by metallic implants. Radiology 1987;164:576-7. 